\title{
Pharmacoeconomic Evaluation of Anti-Hyperlipidemic Agent Fenofibrate
}

\author{
Toru Hayakawa, ${ }^{*, a, b}$ Koichi Shimoyama, ${ }^{b}$ Shigeru SeKiya, ${ }^{b}$ \\ Masatomo SEKIGUCHI, ${ }^{c}$ and Nobuo INOTSUME ${ }^{a}$ \\ Department of Pharmacology and Therapeutics, Hokkaido College of Pharmacy, ${ }^{a}$ 7-1 Katsuraoka-cho, \\ Otaru, Hokkaido 047-0264, Japan and Department of Pharmacyb and Department of \\ Internal Medicine, ${ }^{c}$ Sapporo Kosei Hospital, Higashi-8, Kita-3, \\ Chuo-ku, Sapporo, Hokkaido 060-0033, Japan
}

(Received March 1, 2002; Accepted September 17, 2002)

\begin{abstract}
We evaluated the economic efficiency as well as the clinical effectiveness on serum lipid levels of a change in drug therapy from bezafibrate or a 3-hydroxy-3-methylglutaryl coenzyme A (HMG-CoA) reductase inhibitor to fenofibrate. Subjects were 26 outpatients suffering from type IIb or type IV hyperlipidemia who visited our hospital between October 2000 and January 2001. Medication doses, and serum lipid levels were recorded prior to the change to fenofibrate and at 6 months after the change. Medical costs were also calculated at the same time points. A significant reduction in medical costs of $14.9 \%$ was observed following the change to fenofibrate. Serum lipid levels were not significantly different, although an increase in low density lipoprotein-cholesterol (LDL-cholesterol) was observed in patients changing from the HMG-CoA reductase inhibitor. The actual drug costs were reduced by $21.8 \%$ in the bezafibrate to fenofibrate group and by $23.7 \%$ in the HMG-CoA reductase inhibitor to fenofibrate group. Although the drug costs of changing to fenofibrate decreased significantly, other costs remained almost unchanged.
\end{abstract}

Key words_ - fenofibrate; economics; bezafibrate; hyperlipidemia; 3-hydroxy-3-methylglutaryl coenzyme A reductase inhibitor

\section{INTRODUCTION}

The rising costs of medical care and the increasing financial difficulties of the national health insurance system in Japan have led to a sense of urgency in the medical community. In such a care environment, both the clinical effects and economic efficiency of pharmacotherapy must be considered. This approach to pharmacotherapy has the advantage of reducing drug and hospital administration costs, which leads to a reduction in the costs paid by patients themselves. Although physicians are under increasing pressure to consider patient needs, clinical effects and costs when deciding on a course of treatment, studies of costeffectiveness in medical fields are not well established in Japan. Therefore, a clear need exists for standards by which clinicians may evaluate the therapeutic effects as well as the monetary costs of medication, without neglecting the needs of patients.

Fenofibrate is a drug used to treat hyperlipidemia and is reported to possess an efficacy that is comparable to bezafibrate. ${ }^{1-3)}$ Fenofibrate is reportedly effective at low doses, ${ }^{1-4)}$ and reduces serum uric acid

e-mail: hayakawa@hokuyakudai.ac.jp levels ${ }^{1,5-8)}$ as well as blood sugar levels. ${ }^{9)}$ Furthermore, fenofibrate has been proven effective in treating type IIb hyperlipidemia, in which both total cholesterol and triglyceride increase,,$^{2,10-13)}$ and thus is expected to be prescribed to a wide range of patient groups in an attempt to reduce drug costs (as of 2001: bezafibrate, 121.6 yen/day; fenofibrate, $81.4-122.4$ yen/day).

In the present study, we investigated the potential of fenofibrate to reduce medical costs in Japan, and examined its effectiveness in treating hyperlipidemia.

\section{SUBJECTS AND METHODS}

Subjects were 26 patients suffering from type IIb or type IV hyperlipidemia, who visited the Department of Internal Medicine at Sapporo Kosei Hospital as outpatients between October 1, 2000 and January 31, 2001. All patients were examined by doctors before agreeing to a change in medication from bezafibrate or a 3-hydroxy-3-methylglutaryl coenzyme A (HMG$\mathrm{CoA}$ ) reductase inhibitor to fenofibrate. Oral informed consent was obtained from each patient participating. The present study conformed to the guidelines of the Institutional Review Board for Clinical Study at our hospital. Patient profiles are presented in 
Table 1.

To evaluate clinical effectiveness, serum lipid levels (total cholesterol, low density lipoprotein (LDL)cholesterol, high density lipoprotein (HDL) cholesterol and triglyceride) were measured before the change to fenofibrate and again at 6 months after the change. For LDL-cholesterol, the conversion formula of Friedewald ${ }^{15)}$ was used.

The financial aspects were also evaluated with respect to patient costs, hospital income, and total health insurance payment. Costs were calculated from the total drug administration costs as well as diagnostic and treatment costs (basic treatment costs, administration costs of consultancy, examination and diagnostic costs, injection costs) before the change to fenofibrate and over 6 months after the change. Drug administration costs were divided into actual drug costs and technique costs (prescribing fees, formulating fees, hospital pharmacy fees, and drug information supplying costs). The diagnostic and treatment costs as well as the drug costs partially borne by patients themselves were calculated before and during the 6-month period after the change. Diagnostic and treatment fee tables (calculated from health insurance system regulations) and standard drug costs (official drug price according to the health insurance system) as of October 1, 2000 were used for all cost calculations. The diagnostic and treatment costs borne by patients themselves were calculated based on the ratios set by the insurance of each individual patient. The costs borne by elderly outpatients were calculated as $10 \%$ of total medical costs. The dose of fenofibrate at which the costs prior to the change

Table 1. Patient Background

\begin{tabular}{lc}
\hline \hline No. of cases (male/female) & $26(17 / 9)$ \\
Age (yrs.) & $59.9 \pm 10.7$ \\
BMI $\left(\mathrm{kg} / \mathrm{m}^{2}\right)$ & $25.5 \pm 2.9$ \\
WHO Class & 20 \\
IIb & 6 \\
IV & $20 / 6$ \\
Complicaiton (yes/no) & 11 \\
Diabetes & 9 \\
Hypertension & 3 \\
Ischemic cardiac disorders & 7 \\
Disorders of digestive organs & 3 \\
Hyperuricemia & 3 \\
Plastic surgery disorders & 1 \\
Blood disorders &
\end{tabular}

Table 2. Changes in Doses and Serum Lipid Values before and after Changes of Drugs

\begin{tabular}{|c|c|c|c|}
\hline \multirow{2}{*}{$\begin{array}{l}\text { Changed drug (mg/day) } \\
\text { and serum lipid }{ }^{a)}\end{array}$} & \multirow{2}{*}{$n$} & \multicolumn{2}{|c|}{$\begin{array}{l}\text { Serum lipid concentration } \\
(\mathrm{mg} / \mathrm{dl})^{b)}\end{array}$} \\
\hline & & Before change & After change \\
\hline $\begin{array}{l}\text { Bezafibrate } 400 \text { to } \\
\text { fenofibrate } 200\end{array}$ & 10 & & \\
\hline TC & & $218.4 \pm 14.3$ & $218.6 \pm 14.6$ \\
\hline LDL-C & & $125.7 \pm 13.4$ & $129.2 \pm 17.4$ \\
\hline HDL-C & & $41.5 \pm 2.7$ & $42.3 \pm 2.4$ \\
\hline TG & & $339.5 \pm 53.9$ & $321.8 \pm 54.7$ \\
\hline $\begin{array}{l}\text { Bezafibrate } 400 \text { to } \\
\text { fenofibrate } 100\end{array}$ & 5 & & \\
\hline $\mathrm{TC}$ & & $217.8 \pm 13.8$ & $226.4 \pm 13.7$ \\
\hline LDL-C & & $123.2 \pm 11.5$ & $125.4 \pm 11.9$ \\
\hline HDL-C & & $38.0 \pm 5.0$ & $39.4 \pm 3.7$ \\
\hline TG & & $283.0 \pm 25.2$ & $308.0 \pm 39.2$ \\
\hline $\begin{array}{l}\text { Bezafibrate } 200 \text { to } \\
\text { fenofibrate } 100\end{array}$ & 3 & & \\
\hline $\mathrm{TC}$ & & $203.0 \pm 6.8$ & $209.0 \pm 20.8$ \\
\hline LDL-C & & $119.7 \pm 0.9$ & $109.0 \pm 16.8$ \\
\hline HDL-C & & $35.7 \pm 3.8$ & $37.7 \pm 4.4$ \\
\hline TG & & $342.3 \pm 113.9$ & $295.7 \pm 67.0$ \\
\hline $\begin{array}{l}\text { Total bezafibrate to } \\
\text { fenofibrate }\end{array}$ & 18 & & \\
\hline $\mathrm{TC}$ & & $215.7 \pm 8.7$ & $219.2 \pm 9.3$ \\
\hline LDL-C & & $123.9 \pm 7.5$ & $124.9 \pm 9.6$ \\
\hline HDL-C & & $39.6 \pm 2.1$ & $40.7 \pm 1.8$ \\
\hline TG & & $324.3 \pm 34.5$ & $313.6 \pm 32.8$ \\
\hline $\begin{array}{l}\text { Pravastatin } 10 \text { to } \\
\text { fenofibrate } 200\end{array}$ & 1 & & \\
\hline $\mathrm{TC}$ & & 240 & 228 \\
\hline LDL-C & & 134.8 & 128.2 \\
\hline HDL-C & & 52 & 68 \\
\hline TG & & 266 & 159 \\
\hline $\begin{array}{l}\text { Pravastatin } 10 \text { to } \\
\text { fenofibrate } 100\end{array}$ & 2 & & \\
\hline $\mathrm{TC}$ & & $241 \pm 9.0$ & $258.0 \pm 7.0$ \\
\hline LDL-C & & $155.0 \pm 5.4$ & $182.4 \pm 5.6$ \\
\hline HDL-C & & $37.0 \pm 3.0$ & $40.0 \pm 2.0$ \\
\hline TG & & $245.0 \pm 57.0$ & $178.0 \pm 3.0$ \\
\hline $\begin{array}{l}\text { Simvastatin } 5 \text { to } \\
\text { fenofibrate } 100\end{array}$ & 4 & & \\
\hline TG & & $204.3 \pm 15.5$ & $218.8 \pm 21.5$ \\
\hline LDL-C & & $104.7 \pm 10.2$ & $132.1 \pm 14.7$ \\
\hline HDL-C & & $49.0 \pm 2.9$ & $45.5 \pm 4.1$ \\
\hline TG & & $253.0 \pm 46.9$ & $206.0 \pm 42.3$ \\
\hline $\begin{array}{l}\text { Fluvastatin } 20 \text { to } \\
\text { fenofibrate } 100\end{array}$ & 1 & & \\
\hline $\mathrm{TC}$ & & 218 & 240 \\
\hline LDL-C & & 117.4 & 179.8 \\
\hline HDL-C & & 33 & 39 \\
\hline TG & & 338 & 206 \\
\hline $\begin{array}{l}\text { Total HMG-CoA RI }{ }^{c)} \\
\text { to fenofibrate }\end{array}$ & 8 & & \\
\hline $\mathrm{TC}$ & & $219.6 \pm 9.8$ & $232.4 \pm 11.8$ \\
\hline LDL-C & & $122.6 \pm 9.3$ & $150.1 \pm 11.5^{*}$ \\
\hline HDL-C & & $44.4 \pm 3.0$ & $46.1 \pm 3.8$ \\
\hline TG & & $263.3 \pm 26.6$ & $180.6 \pm 23.2$ \\
\hline Total & 26 & & \\
\hline $\mathrm{TC}$ & & $216.9 \pm 6.6$ & $223.2 \pm 7.3$ \\
\hline LDL-C & & $123.4 \pm 5.7$ & $134.1 \pm 7.7$ \\
\hline HDL-C & & $41.0 \pm 1.8$ & $42.4 \pm 1.7$ \\
\hline TG & & $305.5 \pm 25.5$ & $272.7 \pm 26.5$ \\
\hline
\end{tabular}


become equal to those after the change (threshold value) was calculated. To compare serum lipid levels and changes in costs before and after the change, paired-t test analysis was used, and the level of significance was set at $5 \%$.

\section{RESULTS}

1. Changes in serum lipid values The prescriptions of 18 patients were changed from bezafibrate to fenofibrate, and the prescriptions of 8 patients were changed from the HMG-CoA reductase inhibitor to fenofibrate. No patients were dropped from the study. The mean fenofibrate dose at 6 months after the change was $142.3 \pm 50.3 \mathrm{mg}$. Drug dosage and changes in serum lipid levels over the study period are shown in Table 2 .

No significant difference in serum lipid levels before and after the change was observed overall in the 26 patients. In the 18 of 26 patients that changed from bezafibrate to fenofibrate, no significant difference was seen in serum lipid levels. In the 8 of 26 patients that changed from the HMG-CoA reductase inhibitor, no significant differences were observed in the levels of total cholesterol, HDL-cholesterol or triglyceride, but LDL-cholesterol levels increased significantly $(p<0.05)$.

2. Changes in Costs Changes in cost over the 6-month period are shown in Table 3. The overall costs decreased significantly from 166,939 $\pm 107,116$ yen prior to the change to $142,143 \pm 100,126$ yen $(p<$ 0.05 ) after the change, which is a $14.9 \%$ reduction. Although the costs to the internal medicine department decreased significantly $(p<0.01)$, the costs to other departments remained relatively constant.

Changes in cost were further analyzed by dividing them into 2 categories: diagnostic and medical treatment; and drug administration. No significant difference was observed before and after the change in the diagnostic and medical treatment category. However, costs in the drug administration category were significantly $(p<0.0001)$ lower following the change (Table 4) .

Table 3. Changes in Costs

\begin{tabular}{lcc}
\hline \multicolumn{1}{c}{ Item } & Before changes & After changes \\
\hline Cost $^{a)} / 6$ months $($ yen $)$ & $166,939 \pm 107,116$ & $142,143 \pm 100,126^{*}$ \\
\hline Internal med. costs & $143,237 \pm 97,911$ & $116,675 \pm 98,945^{* * *}$ \\
Costs of other departments $^{b)}$ & $23,702 \pm 43,987$ & $25,468 \pm 42,833$ \\
\hline
\end{tabular}

a) Costs: Total of drug administration costs + diagnostic and tretment costs other than drug administration costs. $b$ ) Costs in other departments: Costs of diagnostic and treatment departments other than internal medicine. ${ }^{*}: p>0.05,{ }^{* * *}: p>0.001$.

Table 4. Change in Drug Administration Cost

\begin{tabular}{lcc}
\hline \multicolumn{1}{c}{ Item } & Before changes & After changes \\
\hline Drug administration cost $^{a} / 6$ months $($ yen $)$ & $91,648 \pm 61,711$ & $71,805 \pm 60,375^{* * * *}$ \\
\hline Drug administration technique cost ${ }^{b)}$ & $5,979 \pm 2,955$ & $5,329 \pm 2,045$ \\
Cost of drugs & $85,669 \pm 12,084$ & $66,476 \pm 11,782^{* * * *}$ \\
$\left.\mathrm{BF} \rightarrow \mathrm{FF}^{c}\right)$ & $85,557 \pm 11,701$ & $66,873 \pm 10,696^{* *}$ \\
$\mathrm{HMG}-\mathrm{CoA} \mathrm{RI} \rightarrow \mathrm{FF}^{d)}$ & $85,920 \pm 30,768$ & $65,583 \pm 31,402^{* * *}$ \\
\hline Drug costs in internal medicine & $75,638 \pm 11,883$ & $56,302 \pm 11,125^{* * * * * *}$ \\
$\mathrm{BF} \rightarrow \mathrm{FF}$ & $72,677 \pm 11,190$ & $53,837 \pm 8,902^{* * *}$ \\
$\mathrm{HMG}-\mathrm{CoA} \mathrm{RI} \rightarrow \mathrm{FF}$ & $82,303 \pm 30,750$ & $61,849 \pm 31,580^{* * * *}$ \\
$\mathrm{Drug}$ costs in other departments & $10,031 \pm 3,182$ & $10,174 \pm 3,756$ \\
$\mathrm{BF} \rightarrow \mathrm{FF}$ & $12,881 \pm 4,324$ & $13,037 \pm 5,210$ \\
$\mathrm{HMG}-\mathrm{CoA} \mathrm{RI} \rightarrow \mathrm{FF}$ & $3,618 \pm 2,623$ & $3,734 \pm 2,624$ \\
\hline
\end{tabular}

a) Drug administration costs: drug administration technique costs + dtug costs. $b$ ) Drug administration technique costs: prescription costs + formulating technique costs + drug information supplying costs. $c$ ) $\mathrm{BF} \rightarrow \mathrm{FF}:$ The groups in which bezafibrate was changed to fenofibrate. $d)$ HMG-CoA RI $\rightarrow$ FF: changed group from HMG-CoA reductase inhibition agent to fenofibrate. ${ }^{* *}: p>0.01,{ }^{* * *}: p>0.001$, ****: $p>0.0001, * * * * * *: p>0.000001$. 
The drug administration category was subdivided into two subcategories (technical costs and actual drug costs) and changes in cost were re-examined. While no significant difference in technical costs was seen, the actual drug costs decreased significantly ( $p$ $<0.0001)$ by $22.4 \%$. A comparative study on actual drug costs based on drug category prior to change demonstrated significant decreases for both the bezafibrate to fenofibrate group, and the HMG-CoA reductase inhibitor to fenofibrate group ( $p<0.01$ and $p<0.001$, respectively) (Table 4 ).

The changes in actual drug cost between departments were studied by comparing costs in the internal medicine department with those in other departments. Although the actual drug costs in the internal medicine department showed a significant decrease $(p$ $<0.000001$ ), no significant difference was observed in the other departments (Table 4).

3. Changes in the Cost Borne by the Patients
The costs borne by patients themselves were reduced significantly from $38,566 \pm 24,480$ yen before the change to $34,757 \pm 26,403$ yen after $(p<0.05)$, which is a reduction of $9.9 \%$ (Table 5 ). These costs were further studied and analyzed by dividing them into diagnostic and treatment expenses and drug costs partially borne by patients. Diagnostic and treatment expenses were significantly reduced $(p<0.05)$, but no change was observed in the drug costs partially borne by patients (Table 5 ).

4. Analysis of the Threshold Values of the Dose per Day of Fenofibrate With the costs of bezafibrate and the HMG-CoA reductase inhibitor administration as criteria, the threshold values for fenofibrate were determined, and the results are shown in Figure 1. For patients who changed from bezafibrate or the HMG-CoA reductase inhibitor to fenofibrate, the cost prior to the change was 166,939 yen, while the cost after was 142,143 yen (average

Table 5. Changes in Amounts to be Borne by Patients

\begin{tabular}{lcc}
\hline \hline \multicolumn{1}{c}{ Item } & Before changes & After changes \\
\hline $\begin{array}{l}\text { Amounts to be borne by patients/6 months } \\
\text { (yen) }\end{array}$ & $38,566 \pm 24,480$ & $34,757 \pm 26,403^{*}$ \\
\hline $\begin{array}{l}\text { Diagnostic and treatment costs to be borne } \\
\text { by patients themselves }\end{array}$ & $31,251 \pm 22,012$ & $28,099 \pm 23,860^{*}$ \\
\begin{tabular}{l} 
Drug costs to be borne by patients \\
\hline
\end{tabular} & $7,314 \pm 5,298$ & $6,658 \pm 4,931$ \\
\hline
\end{tabular}

a) Amounts to be borne by patients temselves: based on the ratio to be borne, set forth by types of insurances. *: $p>0.05$.

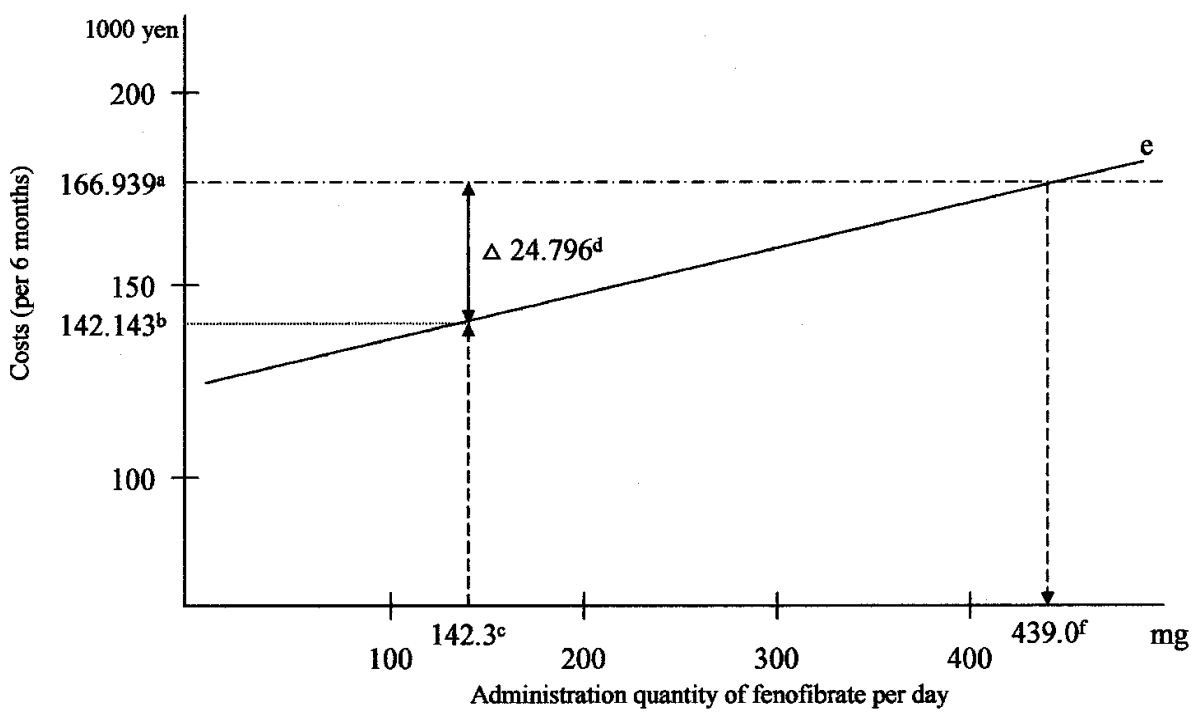

Fig. 1. Threshold Value of Dose of Fenofibrate per Day

a) costs before changes, b) costs after changes, c) mean daily dose of fenofibrate after changes, d) difference in costs between before changes and after changes, e) costs linear line after changes when the daily cost of fenofibrate is increased, f) threshold value (fenofobrate dose at which cost after changes become equivalent, at the costs before changeg as the criterion). 
dose of fenofibrate: $142.3 \mathrm{mg}$ ), a difference of 24,796 yen. The threshold dose (costs before change $=$ costs after change) of fenofibrate was found to be 439.0 $\mathrm{mg}$. The cost of fenofibrate was calculated to be 155,322 yen at $300 \mathrm{mg}$, which is the maximum dose of fenofibrate approved for clinical use.

\section{DISCUSSION}

Following the change from bezafibrate to fenofibrate, no significant change in the serum lipid level was seen. However, a significant increase in LDL-cholesterol was seen in patients changing from the HMG-CoA reductase inhibitor to fenofibrate, although total cholesterol, HDL-cholesterol and triglyceride levels values were not significantly different. HMG-CoA reductase inhibitors are known to reduce total cholesterol and LDL-cholesterol levels, while fibrate drugs reduce triglycerides ${ }^{12,13,16-19)}$ and slightly lower LDL-cholesterol levels. ${ }^{11,19-23)}$ Based on our observations, after a patient is changed from an HMG-CoA reductase inhibitor to fenofibrate, LDL-cholesterol levels should be closely monitored.

In the present study, the cost analysis was performed with regard to the viewpoint of the physician. Changes in cost were calculated from direct expenses for drug treatment. A $14.9 \%$ decrease in costs was observed and was attributed to changes in the drug administration costs. Changes in the number of visits, and the number of examinations, as well as changes in diagnostic and treatment methods, all of which are associated with the change in medication, were thought to influence the costs of diagnosis and treatment, but no change was observed these costs. No change was observed in the technique costs, while the actual drug costs reduced by $22.4 \%$. The formulating technique costs, the prescription and formulating costs, and the drug information supplying costs, which are included in the technique costs, were calculated once a month, at the time of each new prescription, or when any change in dosage occurred. Changes in the number of visits, examination at other departments, and prescription contents were thought to affect technique costs, but no change was observed. The decrease in costs was thought to be because of the reduction in actual drug costs. This is clearly demonstrated by the results of the threshold value analysis, which show a threshold value of $439 \mathrm{mg}$ for fenofibrate. The maximum approved daily dose of fenofibrate is $300 \mathrm{mg}$, and thus treatment with this drug can significantly lower costs.

The observed reduction in costs was primarily centered in the internal medicine department. We initially believed that changes in severity of illness, appearance of new symptoms such as ischemic heart disease and side effects resulting from the change in drugs might affect the costs to other departments but costs to other departments remained unchanged. However, long-term cost evaluation is needed because the above factors are unlikely to be significant within a 6-month period. Furthermore, side effects, changes in severity, and the appearance of new symptoms resulting from the change in medication can only be accurately evaluated in longer term studies.

Changes in actual drug costs after the change to fenofibrate were also centered in the internal medicine department. No changes were observed in actual drug costs in other departments. When we compared drug prices of fenofibrate, HMG-CoA reductase inhibitors and bezafibrate with respect to maximum daily dosage, all the HMG-CoA reductase inhibitors were more expensive than fenofibrate. Bezafibrate and fenofibrate have similar prices based on maximum daily dosage, but several reports have indicated that fenofibrate is equally or more effective at half the dosage of bezafibrate. ${ }^{1-4)}$ In the present study, the clinical effects observed at $156 \mathrm{mg}$ of fenofibrate were similar to those seen at an average bezafibrate dose of $367 \mathrm{mg}$. This increase in potency is likely to be one of the key factors in reducing the actual drug costs to the internal medicine department following the change.

Several studies have investigated the cost effectiveness of HMG-CoA reductase inhibitors, fenofibrate, and other fibrates. In one study, 12 treatment methods for treating hypercholesterol patients were compared, and fluvastatin $(60 \mathrm{mg} /$ day $)$, fenofibrate $(200 \mathrm{mg} /$ day $)$ and simvastatin $(20 \mathrm{mg} /$ day $)$ were reported to be the most cost effective. ${ }^{24)}$ Another study, in which fenofibrate $(200 \mathrm{mg} /$ day $)$ and simvastatin $(20 \mathrm{mg} /$ day $)$ were compared in type $\mathrm{IIb}$ hyperlipidemia treatment, fenofibrate was reported to be more cost effective. ${ }^{25)}$ When fenofibrate, bezafibrate, gemfibrozil, fluvastatin, lovastatin and simvastatin were compared in type IIa and type IIb hyperlipidemia patient groups, simvastatin was the most cost effective in the combined patient group (types IIa and IIb), but in the type IIb patient group, fenofibrate was more cost effective than simvastatin. ${ }^{14)}$ Our results are in agreement with the 
results of these studies and suggest that medical costs could be reduced by prescribing fenofibrate to patients with type IIb or type IV hyperlipidemia.

The reduction in actual drug costs passes savings directly to patients, who are required to pay a portion of incurred medical costs. In particular, significant reductions were observed in the diagnostic and treatment costs borne by patients. Drug selection that considers the monetary cost to the patient is becoming increasingly important because of recent policy changes that make patients liable for a larger portion of their medical expenses. These changes include the January 2001 revision of the health insurance law, which introduced payment based on a fixed percentage of medical costs for aged patients, and the newly established upper limit of maximum costs borne by individuals receiving medical care.

The results of the present study demonstrated a reduction in costs following the change from bezafibrate or the HMG-CoA reductase inhibitor to fenofibrate. This reduction in cost was entirely due to lower drug costs, rather than lower diagnostic and treatment costs or drug administration costs. Hospitals have long benefited from discounted drug prices under the Japanese jurisprudence system, and thus there is a tendency to prescribe more expensive drugs. However, repeated revision of the drug price standard has decreased the adjustment margin for the drug distribution system to $2 \%$. Therefore, utilization of high-priced drugs based on the drug price standard no longer increases hospital income, and hospital management strategy has shifted to improving druguse efficiency by reducing drug costs and limiting the number of drugs available for use in hospitals. The measures discussed in this study would lead to a decrease in income from medical practice, but would not result in a reduction in hospital profits. We expect that reducing drug and administration costs will have numerous positive effects on the long-term management of the medical profession.

\section{CONCLUSION}

Negligible differences in serum lipid levels were demonstrated after the change from bezafibrate to fenofibrate, although a significant increase in LDLcholesterol was seen after the change from the HMGCoA reductase inhibitor to fenofibrate. Changing treatment of type IIb or type IV hyperlipidemia from bezafibrate or the HMG-CoA reductase inhibitor to fenofibrate resulted in effective serum lipid management and reduced total costs by $14.9 \%$, drug costs by $22.4 \%$, and patient costs by $9.9 \%$. These results suggest that, with close monitoring of LDL-cholesterol levels, fenofibrate is a good alternative treatment for hyperlipidemic patients, and significantly reduces medical costs.

\section{REFERENCES}

1) Bastow M. D., Durrington P. N., Ishola M., Metabolism, 37, 217-220 (1988).

2) Klosiewicz-Latoszek L., Szostak W. B., Eur. J. Clin. Pharmacol., 40, 33-41 (1991).

3) Feussner G., Kurth B., Lohrmann J., Eur. J. Med. Res., 2, 165-168 (1997).

4) Tesone P.A., Gladstein J., Acuna A. M., Curr. Med. Res. Opin., 9, 650-657 (1985).

5) Liamis G., Bairaktari E. T., Elisaf M. S., Am. J. Kidney. Dis., 34, 594-595 (1999) .

6) Elisaf M., Tsimichodimos V., Bairaktari E., Siamopoulos K. C., J. Cardiovasc. Pharmacol., 34, 60-63 (1999).

7) Brown W. V., Arteriosclerosis, 6, 670-678 (1986)

8) Serna G., Cadarso C., Clin. Pharmacol. Ther., 66, 166-172 (1999).

9) Tai K., Gotoh Y., Itakura H., Geriat. Med., 33, 295-300 (1994).

10) Bertolini S., Elicio N., Daga A., Degl'Innocenti M.L., Balestreri R., Fusi M. G., Cotta Ramusino A. M., Carozzi A., Eur. J. Clin. Pharmacol., 34, 25-28 (1988).

11) Knopp R. H., Brown W. V., Dijon C. A., Farquhar J. W., Feldman E. B., Goldberg A. C., Grundy S. M., Lasser N. L., Mellies M. J., Palmer R. H., Am. J. Med., 83, 50-59 (1987).

12) Steinmetz A., Schwartz T., Hehnke U., Kaffarnik H., J. Cardiovasc. Pharmacol., 27, 563-570 (1996)

13) Ziegler O., Drouin P., Cardiology, 77, 50-57 (1990).

14) Perreault S., Hamilton V.H., Lavoie F., Grover S., Cardiovasc. Drugs. Ther., 10, 787794 (1996).

15) Friedewald W. T., Levy R. I., Fredrickson D. S., Clin. Chem., 18, 499-502 (1972).

16) Farnier M., Bonnefous F., Debbas N., Irvine A., Arch. Intern. Med., 154, 441-449 (1994).

17) Bruckert E., De Gennes J. L., Malbecq W., 
Baigts F., Clin. Cardiol., 18, 621-629 (1995).

18) Bairaktari E. T., Tzallas C. S., Tsimihodimos V. K., Liberopoulos E. N., Miltiadous G. A., Elisaf M. S., J. Cardiovasc. Risk, 6, 113-116 (1999) .

19) Grundy S. M., Vega G. L., Am. J. Med., 83 (5B), 9-20 (1987).

20) Ginsberg H. N., Am. J. Med., 83 (5B), 66-70 (1987).

21) Stewart J. M., Packard C. J., Lorimer A. R., Boag D. E., Shepherd J., Atherosclerosis, 44,
355-365 (1982).

22) Goldberg A. C., Schonfeld G., Anderson C., Dillingham M. A., Am. J. Med., 83(5B), 6065 (1987).

23) Lewis J. E., Multicenter collaborative Study Group, Pract. Cardiol., 9, 99-118 (1983).

24) Lacour A., Derderian F., LeLorier J., Can. J. Cardiol., 14, 355-361 (1998).

25) Kirchgassler K.U., Schiffner-Rohe J., Stahlheber U., Pharmacoeconomics, 12, 237-246 (1997). 